\title{
Hemangioblastomas aparentemente esporádicos e doença de von Hippel-Lindau não suspeita
}

\author{
Rogelio Iván Ortiz-Velázquez', Jorge Arturo Santos-Franco ${ }^{1}$, Jose Guilherme Mendes \\ Pereira Caldas ${ }^{3}$, Rodrigo Mercado-Pimentel' ${ }^{1}$, Astrid Rasmussen², Rogelio Revuelta1 \\ Departamento de Neurocirurgia e Laboratório de Neurooncogenética do Instituto Nacional de \\ Neurologia e Neurocirurgia Manuel Velasco Suárez, Cidade do México, México \\ Instituto de Radiologia do Hospital das Clínicas da Faculdade de Medicina da Universidade de São Paulo, São Paulo, SP, Brasil
}

\section{RESUMO}

Objetivo: Apresentar os achados associados à doença de von Hippel-Lindau em pacientes portadores de hemangioblastoma do sistema nervoso central, aparentemente esporádico, submetidos a rastreamento (screening) em um centro neurocirúrgico de referência. Métodos: Na base de dados do Instituto Nacional de Neurologia e Neurocirurgia da Cidade do México foram identificados todos os pacientes com diagnóstico histológico confirmado de hemangioblastoma do sistema nervoso central entre janeiro de 1980 e agosto de 2000. Os arquivos médicos, radiológicos e patológicos desses pacientes foram revisados retrospectivamente. Destes, 25 pacientes assintomáticos concordaram em submeter-se ao rastreamento, por meio de exame físico, ultra-sonografia e tomografia axial computadorizada de abdômen, ressonância magnética cranioespinhal e exame oftalmológico. Resultados: $32 \%$ dos pacientes apresentaram policistose, $16 \%$, hemangioblastomas múltiplos e 12\%, carcinomas viscerais. Segundo os critérios de Neumann, logrou-se estabelecer o diagnóstico clínico da doença de von Hippel-Lindau em $60 \%$ dos casos. Conclusão: A presença de lesões assintomáticas nos portadores da doença de von Hippel-Lindau assinala a falta de sensibilidade do exame clínico, fazendo-se necessário o rastreamento de todo paciente portador de hemangioblastomas do sistema nervoso central.

\section{PALAVRAS-CHAVE}

Doença de von Hippel-Lindau. Hemangioblastoma. Diagnóstico, rastreamento.

\section{ABSTRACT}

Apparently sporadic hemangioblastomas and not suspected von Hippel-Lindau disease Objective: To present the findings on patients with central nervous system hemangioblastomas apparently sporadic but associated with von Hippel-Lindau disease, which were analyzed in a third level neurosurgery hospital. Methods: Twenty five clinically asymptomatic patients with the diagnosis of central nervous system hemangioblastoma underwent to a screening for von Hippel-Lindau disease which included physical examination, abdominal ultrasonography and computed axial tomography, craniospinal magnetic resonance imaging and ophthalmologic examination. Results: Thirty two percent of the patients presented multi-organ cystic disease, $16 \%$ multiple hemangioblastomas and $12 \%$ carcinoma. According to the Neumann's criteria, von Hippel-Lindau disease was clinically diagnosed in $60 \%$ of the cases. Conclusion: The presence of asymptomatic lesions in patients with von Hippel-Lindau disease points out the low sensitivity of clinical examination. Therefore, every patient presenting central nervous system hemangioblastoma must be submitted to a screening for this disease.

\section{KEY WORDS}

Hemangioblastoma. von Hippel-Lindau disease. Diagnosis, screening.

1 Neurocirurgião. Departamento de Neurocirurgia do Instituto Nacional de Neurologia e Neurocirurgia Manuel Velasco Suárez, Cidade do México, México.

2 Geneticista do Laboratório de Neurooncogenética do Instituto Nacional de Neurologia e Neurocirurgia Manuel Velasco Suárez, Cidade do México, México.

3 Professor Livre-docente do Departamento de Radiologia da Universidade de São Paulo (USP) e Chefe do Serviço de Neurorradiologia Intervencionista do Hospital das Clínicas da USP. 


\section{Introdução}

No século XIX, diversos relatos apontaram que angiomas retinianos ocasionalmente se associavam às lesões cerebelosas de padrão histológico similar. O oftalmologista alemão Eugene von Hippel foi o primeiro a reconhecer o caráter hereditário de tais angiomas, introduzindo o termo angiomatose retina $e^{21,29}$. Em 1926, o patologista sueco Arvid Lindau correlacionou esses angiomas retinianos e os hemangioblastomas cerebelosos em uma síndrome familiar na qual, ademais, era possível reconhecer outros tipos de tumores e cistos viscerais ${ }^{20,29}$. A revisão de Melmon e Rosen ${ }^{24}$, em 1964, estabeleceu os critérios diagnósticos da doença de von Hippel-Lindau ${ }^{24}$. Essa doença constitui uma síndrome de suscetibilidade familiar ao câncer, apresenta um padrão de herança autossômica dominante, grande variabilidade na sua expressão clínica, penetrância quase completa aos 60 anos e incidência aproximada de $1 / 36.000$ nascidos vivos ${ }^{7,12,14}$. Por outra parte, apesar de os hemangioblastomas esporádicos representarem somente 2,5\% das neoplasias do sistema nervoso central, esses constituem a tumoração intraxial primária da fossa posterior mais comum da idade adulta e até $40 \%$ dos casos associam-se à doença de von Hippel-Lindau (dVHL) $)^{14,19}$. O hemangioblastoma do sistema nervoso central (HSNC) representa a lesão emblemática da doença, domina o quadro clínico e costuma apresentar-se como uma urgência neurocirúrgica, secundariamente ao desenvolvimento de hipertensão intracranial ${ }^{10,20,29}$. No entanto, não existem relatos da prevalência da dVHL entre os pacientes portadores de HSNC em nossa população.

Descrevemos os achados associados ao complexo de VHL em pacientes com diagnóstico de hemangioblastomas aparentemente esporádicos tratados em um centro neurocirúrgico de alta complexidade da Cidade do México.

\section{Material e métodos}

$\mathrm{Na}$ base de dados do Instituto Nacional de Neurologia e Neurocirurgia da Cidade do México, identificaram-se todos os pacientes com diagnóstico histológico confirmado de HSNC, entre janeiro de 1980 e agosto de 2000. Os arquivos médicos, radiológicos e patológicos desses pacientes foram revisados retrospectivamente. Após prévia aprovação da Comissão de Ética e Pesquisa da instituição, procedeu-se a realização de entrevista dos casos clinicamente assintomáticos, propondo-se um rastreamento (screening) com o intuito de identificar lesões silenciosas associadas ao complexo de VHL. O rastreamento consistiu na realização de exame físico, ultra-sonografia e tomografia axial computada de abdômen, imagens por ressonância magnética cranioespinhal e exame oftalmológico.

\section{Resultados}

Identificaram-se 67 casos de hemangioblastomas com diagnóstico confirmado, correspondentes a 1,7\% dos tumores primários do sistema nervoso central, 1,9\% dos tumores intra-axiais intracranianos e $2,3 \%$ dos tumores medulares observados durante o período de revisão. Trinta pacientes foram entrevistados e 25 deles aceitaram a proposta de submeter-se a rastreamento. A idade desses variou entre 16 e 52 anos $(31,3 \pm 9,9)$; a relação entre os gêneros foi de 1:1,5 (homem:mulher); $80 \%$ dos hemangioblastomas apresentaram-se como tumores císticos bem circunscritos com ou sem nódulo mural evidente, oito pacientes (32\%) apresentaram enfermidade cística de múltiplos órgãos, quatro (16\%), hemangioblastomas múltiplos e três $(12 \%)$, tumores viscerais (Tabela 1). Nenhum paciente fez referência à sintomatologia ou mostrou evidência de alterações físicas na exploração clínica. De acordo com os critérios de Neumann, estabeleceu-se o diagnóstico clínico de doença de VHL em $60 \%$ desses casos. Dois pacientes $(8 \%)$ apresentaram antecedentes familiares positivos de hemangioblastomas sem que durante o período de tratamento inicial se estabelecesse o diagnóstico clínico da doença.

\section{Discussão}

A doença de VHL constitui uma síndrome clássica de suscetibilidade familiar ao câncer e se associa a mais de 150 diferentes mutações, deleções ou microdeleções/inserções germinais do gene de $\mathrm{VHL}^{7,21,27}$. $\mathrm{O}$ gene de VHL é um gene supressor do tumor localizado no braço curto do cromossomo 3 (3p25-26) ${ }^{8}$. O produto do gene de VHL ( $\mathrm{pVHL}$ ), uma proteína de 284 aminoácidos e peso molecular de $18-23 \mathrm{Kd}$, regula a angiogênese, a formação da matriz e o ciclo celular. Mutações do gene de VHL permitem a estabilização da transcrição de fatores induzíveis pela hipóxia, e o aumento desses fatores angiogênicos, sob condições de normoxemia, acarreta a aparição das lesões altamente vasculares, patognomônicas da enfermidade ${ }^{27,29}$. Os indivíduos afetados estão em risco de desenvolver 


\begin{tabular}{|c|c|c|c|c|}
\hline \multicolumn{5}{|c|}{$\begin{array}{c}\text { Tabela } 1 \\
\text { Lesões associadas ao complexo de VHL em pacientes assintomáticos previamente } \\
\text { tratados por hemangioblastomas aparentemente esporádicos }\end{array}$} \\
\hline Sexo & Idade (anos) & Localização & Lesões associadas & $\mathbf{A F}$ \\
\hline M & 22 & Bulbomedular & Cistos hepáticos, renais e pancreáticos & + \\
\hline M & 36 & Cerebelo & Cistos renais e pancreáticos & - \\
\hline $\mathrm{F}$ & 16 & Cerebelo & Hemangioblastoma retiniano & - \\
\hline M & 38 & Cerebelo & Cistos pancreáticos & - \\
\hline $\mathrm{F}$ & 34 & Cerebelo & Cistos renais e pancreáticos & - \\
\hline $\mathrm{F}$ & 40 & Cerebelo & Feocromocitoma & - \\
\hline $\mathrm{F}$ & 32 & Cerebelo & Tumor pancreático & - \\
\hline M & 27 & Cerebelo & Carcinoma renal & - \\
\hline $\mathrm{F}$ & 24 & Cerebelo & Cistos renais e pancreáticos & - \\
\hline $\mathrm{F}$ & 29 & Cerebelo & Hemangioblastomas sincrônicos & - \\
\hline $\mathrm{F}$ & 17 & Cerebelo & Hemangioblastoma retiniano & - \\
\hline M & 31 & Cerebelo & Cistos renais e pancreáticos & - \\
\hline $\mathrm{F}$ & 52 & Cerebelo & Cistos hepáticos, renais e pancreáticos & - \\
\hline M & 27 & Cerebelo & Hemangioblastomas metacrônicos & + \\
\hline $\mathrm{F}$ & 45 & Supratentorial & Cistos renais e pancreáticos & - \\
\hline
\end{tabular}

AF: Antecedentes familiares de hemangioblastoma

seis diferentes tipos de lesões cardinais: hemangioblastomas do SNC e retinianos, cistos ou carcinomas renais e pancreáticos, feocromocitomas e tumores do saco endolinfático ${ }^{7,8,12,14,18-21,27-29}$. Os critérios diagnósticos, segundo Melmon e Rosen, modificados por Neumann ${ }^{27-}$ ${ }^{30}$, incluem hemangioblastomas múltiplos ou um hemangioblastoma mais uma lesão visceral maior.

$\mathrm{Na}$ presença de história familiar positiva, que é possível reconhecer, segundo a literatura mundial ${ }^{12,18,27}$, em $80 \%$ dos casos, uma lesão maior é suficiente para estabelecer o diagnóstico clínico.

Os pacientes com lesões típicas, porém com história familiar negativa, deverão ser considerados portadores de uma mutação "de novo"20,21,27,29.

Diante de hemangioblastomas aparentemente esporádicos existem pelo menos duas justificativas para o estabelecimento de um programa de rastreamento da dVHL (Tabela 2) ${ }^{4}$. Inicialmente, a identificação de pacientes portadores da mutação permitiria uma adequada orientação genética e, em segundo lugar, a detecção precoce de lesões associadas ao complexo de VHL, responsáveis da morbimortalidade tardia, em pacientes ainda assintomáticos, provavelmente permitiria estender a sobrevida e melhorar a qualidade desta ${ }^{4,6,16}$.

Neste estudo, ao tempo do diagnóstico inicial, no subgrupo de pacientes submetidos a rastreamento, somente dois reconheceram antecedentes familiares positivos, mas o segundo interrogatório e a busca de lesões associadas à doença permitiram identificar um padrão de herança autossômica dominante em $93 \%$ dos

\begin{tabular}{lc}
\hline & Tabela 2 \\
& Protocolo de rastreamento \\
\hline Semestral & Bianual \\
Exame neurológico & RM cranioespinal \\
Exame neurootológico & TC abdominal \\
Exame neurooftalmológico & \\
Ultra-sonografia abdominal & \\
Determinação de catecolaminas urinárias & \\
\hline
\end{tabular}

casos positivos ao rastreamento. Essas diferenças dependem da abordagem diagnóstica pré-operatória e da omissão do rastreamento pós-cirúrgico, pois somente em $26 \%$ dos casos a suspeita diagnóstica pré-operatória incluiu a possibilidade de um hemangioblastoma, sendo a maioria dos casos submetida a tratamento cirúrgico com o diagnóstico de neurocisticercose ou astrocitoma cístico e em nenhum deles foi proposto o escrutínio depois de corroborado o diagnóstico histológico de hemangioblastoma. Esses resultados confirmam, em concordância com a literatura mundial ${ }^{10,15,35}$, que a principal dificuldade no manejo da dVHL é o subdiagnóstico.

Em contraste com a homogeneidade do locus genético, a expressão clínica variável da dVHL freqüentemente atrasa e confunde o diagnóstico ${ }^{9}$. Apesar de um órgão afetado poder apresentar lesões múltiplas, que oscilam entre cistos benignos, tumores vasculares ou 
carcinomas, e de a penetrância variável gerar diversos graus de afecção, desde a síndrome completa até os hemangioblastomas solitários familiares considerados como formas frustradas da doença ${ }^{10,15,35}$, deverá considerar-se o hemangioblastoma do sistema nervoso central como a lesão característica e a manifestação mais comum da dVHL ${ }^{10,15,20,21,29,40}$. O hemangioblastoma pode ser isolado e a única expressão da enfermidade, a despeito da característica afecção visceral de múltiplos órgãos. Em populações anglo-saxônicas, 15\% a 40\% dos hemangioblastomas cerebelosos e $20 \%$ a $60 \%$ dos hemangioblastomas espinhais aparentemente esporádicos se associam à dVHL $10,15,20,21,29,40$.

No conhecimento dos autores, esta série representa a maior entre as séries da América Latina. Contudo, a alta prevalência encontrada da dVHL $(60 \%)$ representa um evidente viés de referência e seleção da amostra.

As localizações habituais do hemangioblastoma do sistema nervoso central incluem o cerebelo, o tronco cerebral e a medula espinhal. Em comparação com o relatado na literatura ${ }^{10,15,20,21,29,35}$, somente $4,5 \%$ de nossos casos apresentaram localização extracerebelar (Figura 1). Da mesma forma, e em concordância com outras séries ${ }^{10,15,20,21,29,35}, 80 \%$ dos hemangioblastomas neste estudo se apresentaram como tumores císticos bem circunscritos com ou sem nódulo mural evidente (Figura 2). O tratamento de eleição consiste na remoção cirúrgica; no entanto, no âmbito da dVHL, o desenvolvimento de hemangioblastomas múltiplos sincrônicos e metacrônicos constitui o principal desafio terapêutico ${ }^{32,41}$ (Figura 3).

Entre os pacientes com dVHL, $70 \%$ desenvolvem hemangioblastomas de retina antes da sétima década da vida ${ }^{11}$. Por outra parte, a prevalência de dVHL entre os pacientes com hemangioblastomas retinianos aparentemente esporádicos é de $30 \%$ aos 60 anos $^{26}$. Os hemangioblastomas retinianos costumam ser multifocais, bilaterais e recorrentes em $50 \%$ dos $\operatorname{casos}^{11,26}$. Localizam-se, preferentemente, na retina periférica, não obstante, possam afetar a mácula e a papila. Entre os pacientes do estudo, $13 \%$ apresentaram lesões retinianas no momento do diagnóstico. Freqüentemente, manifestam-se mediante glaucoma ou desprendimento de retina e, já que o desprendimento comumente ocorre sem sintomas prévios ou fatores desencadeantes identificáveis, essa forma de apresentação constitui o mais poderoso argumento em favor do rastreamento oftalmológico de rotina em pacientes portadores ou em risco de padecer da dVHL $\mathrm{dVH}^{11,20,21,26,29,36}$.

Um terço dos pacientes com dVHL apresenta lesões renais múltiplas e bilaterais ao momento do diagnóstico ${ }^{3,5,33}$. Neste estudo, $46 \%$ dos indivíduos apresentaram cistos renais. Essas lesões podem simular outras doenças císticas do rim, tais como a doença
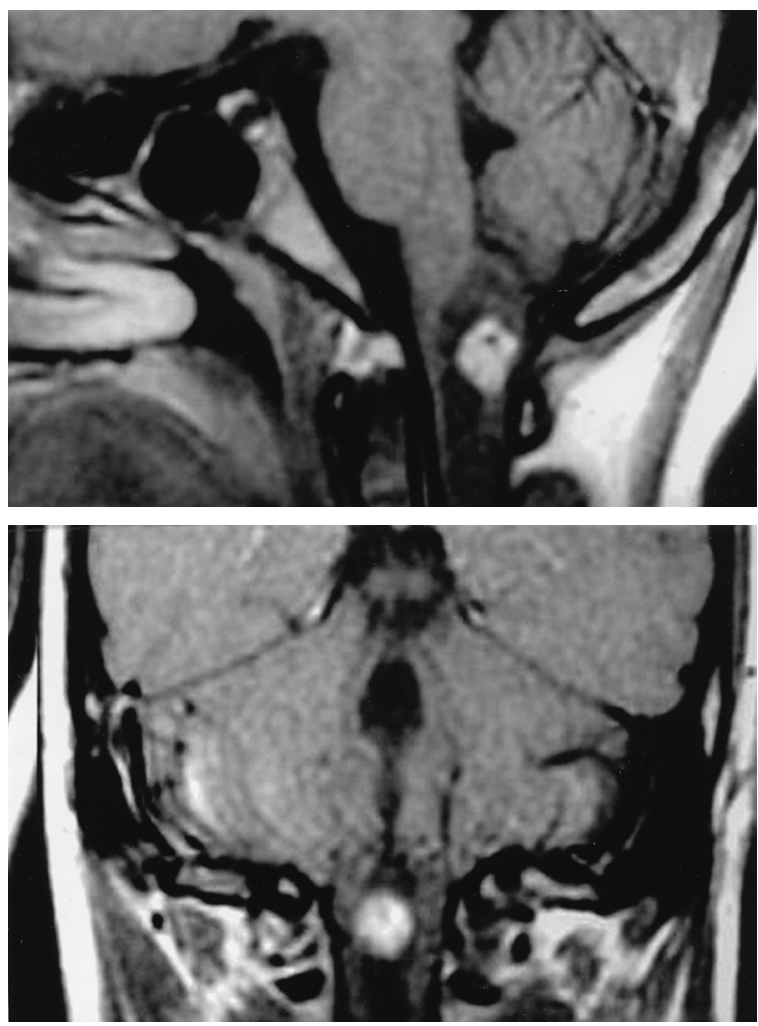

Figura 1 - Imagens sagital coronal de RM ponderada em T1 após infusão venosa do gadolínio-DTPA, identificando-se tumor misto (cístico-sólido) com intenso realce do componente sólido, compativel com hemangioblastoma da união bulbomedular.

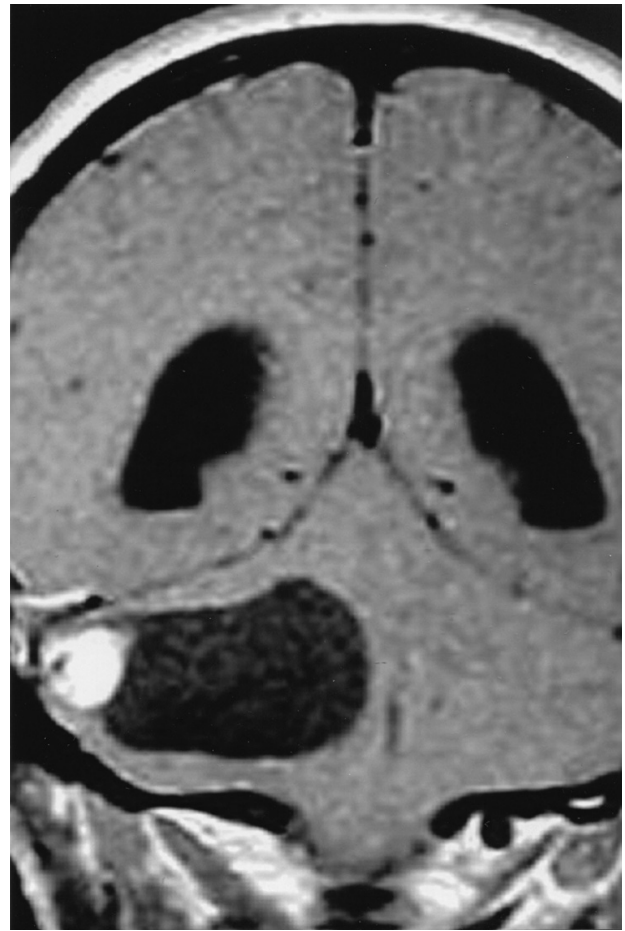

Figura 2 - Imagem coronal de RM ponderada em T1 após infusão venosa do gadolínio-DTPA, onde se observa lesão compativel com hemangioblastoma cerebeloso de características típicas (cisto com nódulo mural). 


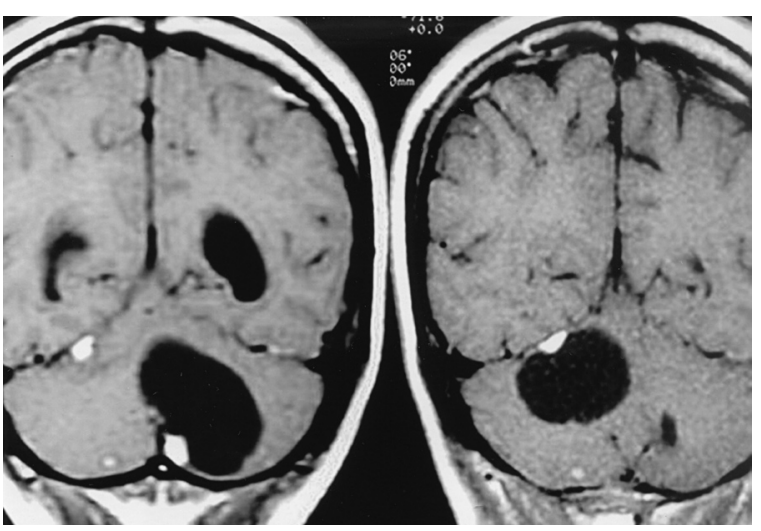

Figura 3 - Esquerda: imagem coronal de RM ponderada em T1 após infusão venosa do gadolínio-DTPA. Observam-se diversas lesões compativeis com hemangioblastomas cerebelosos sincrônicos. Uma delas com características típicas (cisto com nódulo mural). Direita: quatro anos após a ressecção do hemangioblastoma cerebeloso esquerdo, a lesão hemisférica direita desenvolveu componente cístico.

policística renal ${ }^{17}$ (Figura 4). Os tumores sólidos do rim na dVHL, invariavelmente, representam carcinomas de células claras e ocorrem em $25 \%$ a $45 \%$ dos portadores ${ }^{17,22,25,30,31,34,38,39}$. O aperfeiçoamento do rastreamento e o tratamento eficaz dos hemangioblastomas e o feocromocitoma, potencialmente fatais em pacientes jovens, têm permitido que o câncer renal se transforme na principal causa de morte, um terço das quais secundária à doença metastática ${ }^{3,30,31,33,34,37,38}$. A prevalência de dVHL em séries de câncer renal aparentemente esporádico é de 1,6\% $\%^{34,37,38,39}$.

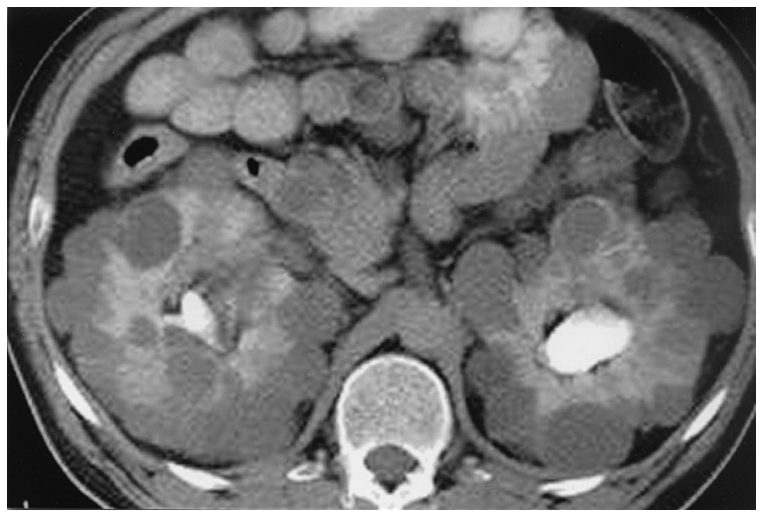

Figura 4-TC de abdômen com contraste oral e intravenoso que demonstra múltiplos cistos renais bilaterais distribuídos em forma difusa. Essas imagens são indistinguiveis das observadas em pacientes com doença renal policística.

Até $20 \%$ dos feocromocitomas associam-se a dVHL ${ }^{1,28}$. Não obstante, o feocromocitoma apresentase em $5 \%$ a $60 \%$ das famílias portadoras da enfermi- dade, o que reflete as grandes diferenças fenotípicas interfamiliares ${ }^{1,20,21,28,29}$. O feocromocitoma pode ser unilateral, multifocal, extra-adrenal e a única manifestação da doença ${ }^{28}$. A tomografia computadorizada de abdômen, realizada freqüentemente como o primeiro estudo de rastreamento em pacientes com suspeita de dVHL, habitualmente permite a detecção inicial dessas lesões, que usualmente se mantêm quiescentes e onde os estudos urinários e as gamagrafias podem resultar normais ${ }^{1}$, comportamento exibido por um dos casos de nosso estudo (Figura 5).
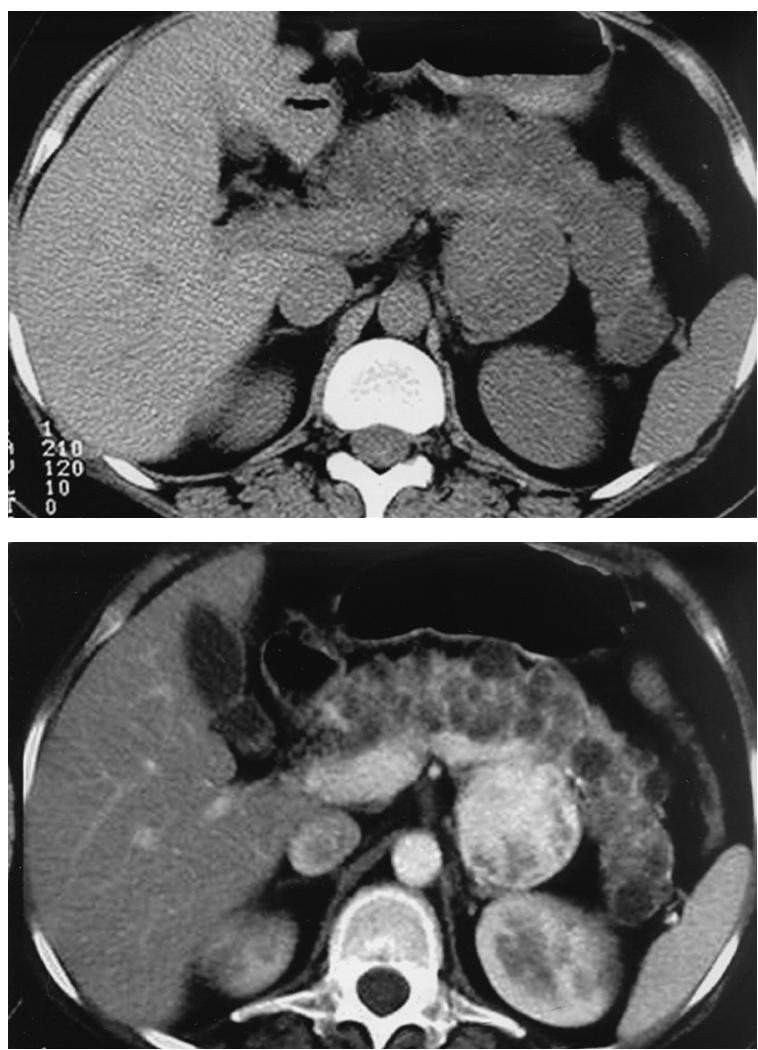

Figura 5 - TC de abdômen em fase simples (acima) e fase contrastada (abaixo). Observa-se a presença de tumoração sólida com realce intenso dependente da glândula supra-renal esquerda, compativel com feocromocitoma. Visualizam-se, ainda, múltiplos cistos pancreáticos de aspecto simples, em todos os segmentos da dita glândula.

A manifestação pancreática mais freqüente do complexo de VHL são os cistos simples, presentes em $53 \%$ dos sujeitos positivos ao rastreamento durante este estudo. Entretanto, a prevalência reportada oscila entre $3 \%$ e $93 \%{ }^{13}$. Os tumores neuroendócrinos das ilhotas representam carcinomas que aparecem de maneira independente à doença cística do pâncreas ${ }^{2,13}$. O diagnóstico presuntivo realiza-se mediante estudos de imagem, nos quais, caracteristicamente, aparecem como lesões sólidas que se intensificam nas fases com contraste ${ }^{1-4,9,20,21,29}$ (Figura 6). 


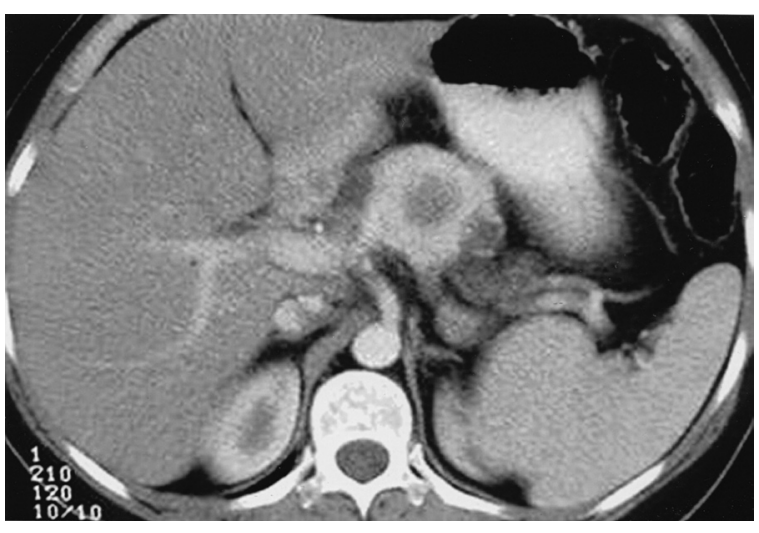

Figura 6-TC após contraste oral e endovenoso demonstra lesão nodular do corpo pancreático, reforçando intensamente na sua periferia e zona central sem realce, compatível com tumor das ilhotas pancreáticas.

Os tumores do saco endolinfático apresentam-se em até $10 \%$ dos portadores ${ }^{23}$ e localizam-se na região compreendida entre a dura-máter e a parede posteromedial da porção petrosa do osso temporal, no final do saco endolinfático. Seu crescimento local com compressão do cerebelo e obliteração do ângulo pontocerebeloso pode ocasionar confusão com lesões características dessa localização, a saber: schwannoma vestibular ou meningioma petroso. Apesar da busca dirigida, nenhum dos pacientes desta série apresentou evidência de lesões da região do saco endolinfático.

Assim, a existência de lesões assintomáticas entre os portadores da dVHL assinala a falta de sensibilidade do exame clínico. Conseqüentemente, todo paciente portador de hemangioblastomas deverá submeter-se a rastreamento, mesmo na ausência de história familiar positiva. Por outra parte, a identificação de um paciente comprometido pela enfermidade exige o rastreamento de todos os membros da família potencialmente afetados, processo de custo elevado. A esse respeito, estandardizar a técnica de diagnóstico molecular permite otimizar os recursos diagnósticos e terapêuticos, além de caracterizar as mutações do gene de VHL próprias da população em estudo. Após os resultados desse estudo, o Instituto Nacional de Neurologia e Neurocirurgia da Cidade do México incorporou o diagnóstico molecular da dVHL em todo paciente portador de hemangioblastoma do sistema nervoso central.

\section{Conclusão}

A presença de lesões assintomáticas nos portadores da doença de von Hippel-Lindau assinala a falta de sensibilidade do exame clínico, fazendo-se necessário o rastreamento de todo paciente portador de hemangioblastomas do sistema nervoso central.

\section{Referências}

1. APRILL BS, DRAKE AJ III, LASSETER DH, SHAKIR KMM: Silent adrenal nodules in von Hippel-Lindau disease suggest pheochromocytoma. Ann Intern Med 120:485-87, 1994.

2. BINKOVITZ LA, JOHNSON CD, STEPHENS DH: Islet cell tumors in von Hippel-Lindau disease: increase prevalence and relationship to the multiple endocrine neoplasms. AJR 155:501-5, 1990.

3. CHAUVEAU D, DUVIC C, CHRÉTIEN Y, PARAF F, DROZ $D, M E L K I P$ et al.: Renal involvement in von Hippel-Lindau disease. Kidney Int 50:944-51, 1996.

4. CHOYKE PL, FILLING-KATZ MR, SHAWKER TH, GORIN $M B$, TRAVIS WD, CHANG R et al.: von Hippel-Lindau disease: radiologic screening for visceral manifestations. Radiology 174:815-20, 1990.

5. CHOYKE PL, GLENN GM, WALTER MM, ZBAR B, WEISS $\mathrm{GH}$, ALEXANDER RB et al.: The natural history of renal lesions in von Hippel-Lindau disease: a serial CT imaging study in 28 patients. AJR 159:1229-34, 1992.

6. CHOYKE PL, GLENN GM, WALTER MM, PATRONAS NJ, LINEHAN WM, ZBAR B. von Hippel-Lindau disease: genetic, clinical and imaging features. Radiology 194:629-42, 1995.

7. COUCH V, LINDOR N, KARNES S, MICHELS V: von HippelLindau disease. Mayo Clin Proc 75:265-72, 2000.

8. LATIF F, TORY K, GNARRA J, YAO M, DUH FM, ORCUTT $M L$ et al.: Identification of the von Hippel-Lindau disease tumor supresor gene. Science 260:1317-20, 1993.

9. FILL WL, LAMIELL JM, POLK NO: The radiographic manifestations of von Hippel-Lindau disease. Radiology 133:289-95, 1979.

10. FILLING-KATZ MR, CHOYKE PL, OLDFIELD E, CHARNAS L, PATRONAS NJ, GLENN GM et al.: Central nervous system involvement in von Hippel-Lindau disease. Neurology 41:41-6, 1991.

11. GROSSNIKLAUS HE, THOMAS JW, VIGNESWARAN N, JARRETT WH: 3rd. Retinal hemangioblastoma. A histologic, immunohistochemical, and ultrastructural evaluation. Ophthalmology 99:140-5, 1992.

12. HORTON WA, WONG V, ELDRIDGE R: Clinical and pathological manifestations in nine families with 50 affected members. Arch Intern Med 136:769-7, 1976.

13. HOUGHDM, STEPHENS DH, JOHNSON CD, BINKOVITZLA: Pancreatic lesions in von Hippel-Lindau disease: prevalence, clinical significance and CT findings. AJR 162:1091-94, 1994.

14. HUBSCHMANN OR, VIJAYANATHAN T, COUNTREE RW: von Hippel-Lindau disease with multiple manifestations: diagnosis and management. Neurosurgery 8:92, 1981.

15. HUSON SM, HARPER PS, HOURIHAN MD, COLE G, WEEKS RD, COMPSTON DA: Cerebellar haemangioblastoma and von Hippel-Lindau disease. Brain 109:1297-307, 1986.

16. KARSDORP N, ELDERSONA, WITTEBOL-POST D, HENÉ RJ, VOS J, FELDBERG MA: von Hippel-Lindau disease: new strategies in early detection and treatment. Am J Med 97:158-8, 1994.

17. LAMIELL JM, STOR RA, HSIA YE: von Hippel-Lindau disease simulating polycystic kidney disease. Urology 15:287-90, 1980. 
18. LAMIELL JM, SALAZAR FG, HSIA YE: von Hippel-Lindau disease affecting 43 members of a single kindred. Medicine (Baltimore) 68:1-29, 1989.

19. MACK E: Neurologic tumors. The genetics basis of neurosurgical disorders. In Youmans JR (ed): Neurological surgery. 7th ed. Philadelphia, WB Saunders, 1995, v. II, part VI, chapter 31, pp. 1071-86.

20. MAHER ER, YATES JR, HARRIES R, BENJAMIN C, HARRIS R, MOORE AT et al.: Clinical features and natural history of von Hippel-Lindau disease. Q J Med 77:1151-63, 1990.

21. MAHER E, KAELIN JR WG: von Hippel-Lindau disease. Medicine (Baltimore) 76:381-91, 1997.

22. MALEK RS, OMESS PJ, BENSON RC, ZINCKE H: Renal cell carcinoma in von Hippel-Lindau syndrome. Am J Med 82:236-8, 1987.

23. MANSKI TJ, HEFFNER DK, GLENN GM, PATRONAS NJ, PIKUS AT, KATZ D et al.: Endolymphatic sac tumors. A source of morbid hearing loss in von Hippel-Lindau disease. JAMA 277:1461-6, 1997.

24. MELMON KL, ROSEN SW: Lindau's disease. Review of the literature and study of large kindred. Am J Med 24:350-7, 1964.

25. MILLER DL, CHOYKE PL, WALTHER MM, DOPPMAN JL, KRAGEL PJ, WEISS GH et al.: von Hippel-Lindau disease: inadequacy of angiography for identification of renal cancers. Radiology 179:833-6, 1991.

26. MOORE AT, MAHER ER, ROSEN P, GREGOR Z, BIRD AC: Ophthalmological screening for von Hippel-Lindau disease. Eye 5:723-8, 1991.

27. NEUMANN HP, WIESTLER OD: Clustering of features of von Hippel-Lindau syndrome: evidence for a complex genetic locus. Lancet 337:1052-4, 1991.

28. NEUMANN HP, BERGER DP, SIGMUND G, BLUM U, SCHMIDT D, PARMER RJ, et al.: Pheochromocytomas, multiple endocrine neoplasia type 2, and von Hippel-Lindau disease. N Engl J Med 329:1531-8, 1993.

29. NEUMANN HP, LIPS CJ, HSIA YE, ZBAR B: von HippelLindau syndrome. Brain Pathol 5:181-93, 1995.

30. NEUMANN H, ZBAR B: Renal cysts, renal cancer and von Hippel-Lindau disease. Kidney Int 51:16-26, 1997.

31. NEUMANN HP, BENDER BU, BERGER DP, LAUBENBERGER J, SCHULTZE-SEEMANN W, WETTERAUER U: Prevalence, morphology and biology of renal cell carcinoma in von Hippel-Lindau disease compared to sporadic renal cell carcinoma. J Urol 160:1248-54, 1998.

32. PAGE KA, WAYSON K, STEINBERG GK, ADLER JR: Stereotaxic radiosurgical ablation: an alternative treatment for recurrent and multifocal hemangioblastomas. A report of four cases. Surg Neurol 40:424-8, 1993.
33. PARAF F, CHAUVEAU D, CHRÉTIEN Y, RICHARD S, GRÜNFELD JP, DROZ D: Renal lesions in von Hippel-Lindau disease: immunohistochemical expression of nephron differentiation molecules, adhesion molecules and apoptosis proteins. Histopathology 36:457-65, 2000.

34. POSTON CD, JAFFE GS, LUBENSKY IA, SOLOMON D, ZBAR B, LINEHAN WM et al.: Characterization of the renal pathology of a familial form of renal cell carcinoma associated with von Hippel-Lindau disease: clinical and molecular genetic implications. J Urol 153:22-6, 1995.

35. RICHARDS S, MARTIN S, DAVID P, DECQ P: Maladie de von Hippel-Lindau et hémangioblastomes du système nerveux central: Applications cliniques des progrès génétiques. Neurochirurgie 44:258-66, 1998.

36. SALAZAR FG, LAMIELL JM: Early identification of retinal angiomas in a large kindred von Hippel-Lindau disease. Am J Ophthalmol 89:540-5, 1980.

37. STEINBACH F, NOVICK AC, ZINCKE H, MILLER DP, WILLIAMS RD, LUND G, et al.: Treatment of renal cell carcinoma in von Hippel-Lindau disease: a multicenter study. J Urol 153:1812-6, 1995.

38. WALTHER MM, LUBENSKY IA, VENZON D, ZBAR B, LINEHAN WM: Prevalence of microscopic lesions in grossly normal renal parenchyma from patients with von HippelLindau disease, sporadic renal cell carcinoma and no renal disease: clinical implications. J Urol 154:2010-4, 1995.

39. WALTHER MM, LINEHAN WM: Nephron sparing surgery for renal cell carcinoma in von Hippel-Lindau disease. J Urol 156(2 pt 1):480-1, 1996.

40. YAMASHITA J, HANDA H, KIM C, KIM S: Familial occurrence of cerebellar hemangioblastomas-analysis of five families. Neurosurgery 11:761-3, 1982.

41. Young S, Richardson AE. Solid haemangioblastomas of the posterior fossa: radiological features and results of surgery. J Neurol Neurosurg Psychiatry 50:155-8, 1987.

Original recebido em fevereiro de 2008

Aceito para publicação em junho de 2008

\section{Endereço para correspondência}

Rogelio Iván Ortiz-Velázquez

Av. Dr. Enéas de Carvalho Aguiar, $255-3^{\circ}$ andar

05403-001 - São Paulo, SP

E-mail: rivanov72@usp.br 